\title{
Properties of Nanostructure Bismuth Telluride Thin Films Using Thermal Evaporation
}

\author{
Swati Arora, ${ }^{1,2}$ Vivek Jaimini, ${ }^{1}$ Subodh Srivastava, ${ }^{2}$ and Y. K. Vijay ${ }^{2}$ \\ ${ }^{1}$ Swami Keshvanand Institute of Technology, Management \& Gramothan, Jaipur, India \\ ${ }^{2}$ Vivekananda Global University, Jaipur, India \\ Correspondence should be addressed to Swati Arora; aroraswati14@gmail.com
}

Received 22 December 2016; Revised 28 February 2017; Accepted 2 March 2017; Published 22 March 2017

Academic Editor: Andrey E. Miroshnichenko

Copyright (c) 2017 Swati Arora et al. This is an open access article distributed under the Creative Commons Attribution License, which permits unrestricted use, distribution, and reproduction in any medium, provided the original work is properly cited.

\begin{abstract}
Bismuth telluride has high thermoelectric performance at room temperature; in present work, various nanostructure thin films of bismuth telluride were fabricated on silicon substrates at room temperature using thermal evaporation method. Tellurium (Te) and bismuth (Bi) were deposited on silicon substrate in different ratio of thickness. These films were annealed at $50^{\circ} \mathrm{C}$ and $100^{\circ} \mathrm{C}$. After heat treatment, the thin films attained the semiconductor nature. Samples were studied by X-ray diffraction (XRD) and scanning electron microscopy (SEM) to show granular growth.
\end{abstract}

\section{Introduction}

Bismuth telluride $\left(\mathrm{Bi}_{2} \mathrm{Te}_{3}\right)$ is a semiconductor with an indirect bulk energy band gap of $0.165 \mathrm{eV}$. It is layered semiconductor material having trigonal structure with high melting point $\left(585^{\circ} \mathrm{C}\right)$ and a density of $7.7 \mathrm{~g} / \mathrm{cm}^{3}$; also it is the heaviest stable binary compound [1]. These interesting properties grabbed the researchers' attention for its wide range application in the field of thermoelectric material [2]. Since last decades, efforts are in progress to enhance the properties of these materials using different approaches and one of the fruitful approaches is to synthesize it in nanoscale [3]. Till today, many methods have been discussed to prepare nanostructure bismuth telluride with various sizes and shapes such as microwave assisted method, mechanical alloying, ball milling, hot pressing, spark plasma sintering, and wet chemical method [4].

It is an attractive thermoelectric material with the highest figure of merit $(Z T=0.68)$ at room temperature in its bulk. Nanostructuring layered super lattice structure of $\mathrm{Bi}_{2} \mathrm{Te}_{3}$ produces a device within which there is good electrical conductivity but perpendicular to which thermal conductivity is poor. Bismuth telluride compounds are usually obtained with directional solidification from melt or powder metallurgy processes [5]. Materials produced with these methods have lower efficiency than single crystalline ones due to the random orientation of crystal grains, but their mechanical properties are superior and the sensitivity to structural defects and impurities is lower due to high optimal carrier concentration. The required carrier concentration is obtained by choosing a nonstoichiometric composition, which is achieved by introducing excess bismuth or tellurium atoms to primary melt or by dopant impurities. Some possible dopants are halogens and group IV and V atoms. Due to the small band gap $(0.16 \mathrm{eV}), \mathrm{Bi}_{2} \mathrm{Te}_{3}$ is partially degenerate and the corresponding Fermi level should be close to the conduction band minimum at room temperature. The size of the band gap means that $\mathrm{Bi}_{2} \mathrm{Te}_{3}$ has high intrinsic carrier concentration. $\mathrm{Bi}_{2} \mathrm{Te}_{3}$ is a narrow band gap semiconductor [2].

In this paper, we have synthesized the compound thin film by depositing the elemental layers and thermal annealing. Typical measurement of different samples having different thickness shows similar behaviour of resistivity measurement. However, the data corresponding to the thickness of $500 \mathrm{~nm}$ are presented here.

\section{Experimental Details}

Tellurium (Te) was purchased from Koch-Light Laboratories Ltd., Colnbrook, Berkshire, England. Bismuth (Bi) was purchased from Sigma-Aldrich laboratories. Thin film of Te 
and $\mathrm{Bi}$ has been deposited using thermal evaporation method on a properly cleaned silicon substrate (n type) of $1 \times 1 \mathrm{~cm}^{2}$ dimension. Initially, clean the tungsten boat and chamber by acetone. The tellurium (Te) and bismuth (Bi) powders having $99.99 \%$ purity were placed in different tungsten boats and start the pumping unit to create the high vacuum in the chamber, respectively. After reaching the high vacuum $\left(10^{-5}\right.$ torr $)$ in the chamber, Te and Bi were heated indirectly by passing the current slowly to the electrodes. $\mathrm{Bi}$ and then Te of $1: 1$ ratio total thickness $(200 \mathrm{~nm}, 500 \mathrm{~nm}, 750 \mathrm{~nm}$, and $1000 \mathrm{~nm}$ ) have been deposited on silicon substrate. We expected that the number of atoms present for the mixing will be reciprocal to the ratio of their mass number (for bismuth (208) and for tellurium (127)) to synthesize $\mathrm{Bi}_{2} \mathrm{Te}_{3}$ phase (without changing density parameter in quartz crystal monitor). Thickness of the $\mathrm{Bi}$ and $\mathrm{Te}$ layer on substrate was controlled using quartz crystal monitor ("Hind Hivac" Digital Thickness Monitor Model, DTM-101).

The surface morphologies of thin films were analysed by Nova NanoSEM 450 scanning electron microscope (SEM). Structural characterization was done using PANalytical's X'Pert PRO manufactured X-ray diffraction (XRD) unit and electrical properties were studied by Four Probe ES-246 setup manufactured by OMEGA. Each sample was studied by X-ray diffraction (XRD) and scanning electron microscopy (SEM) to obtain comprehensive and consistent micro structural information [6].

\section{Results and Discussion}

3.1. Electrical Properties. We observed that without annealing thin film shows the typical metallic nature and shows high conducting without any junction effect. This can be also seen in Figure 1. The $V-I$ curves show the typical linear behaviour of thin films of different thickness $(200 \mathrm{~nm}, 500 \mathrm{~nm}, 750 \mathrm{~nm}$, and $1000 \mathrm{~nm}$ ) at room temperature.

This shows that after annealing the tellurium partially diffuses into bismuth and there would be mixing in the interface and makes compound films. This can be also seen by the $V$ - $I$ curve in Figure 2 of $500 \mathrm{~nm}$ thickness annealed at $100^{\circ} \mathrm{C}$ which shows typical nonlinear curves due to granular uniformity of the surface of thin film. Junction has formed at the interface of bismuth and tellurium. Thin film indicates that after heat treatment the thin films attained the semiconductor nature and hence conductivity is decreased and band gap increases.

3.2. Structural Properties of the Thin Film. The structural properties of the thin films have been characterized by recorded XRD pattern of thin films with or without annealing [7]. Figure 3 shows the XRD pattern of $500 \mathrm{~nm}$ thin film. It indicates that characteristics of XRD peaks corresponding to bismuth and tellurium have been observed at specific $2 \theta$ angle corresponding to the orthorhombic face of thin films [8].

In this work, it has been demonstrated that as $\mathrm{Bi}$ and $\mathrm{Te}$ are deposited on $\mathrm{Si}$ substrate, all peaks of $\mathrm{Si}$ and $\mathrm{Bi}$ are shown in XRD and no peak of Te is there due to noncrystalline behaviour of Te. After annealing, Te is easily

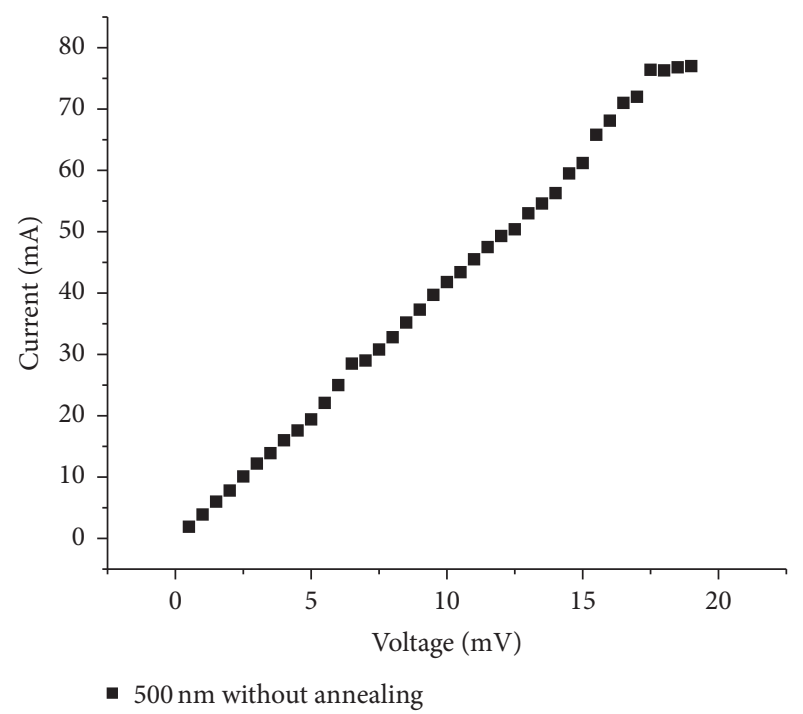

FIgURE 1: Dependence of voltage on current for a sample not subjected to annealing.

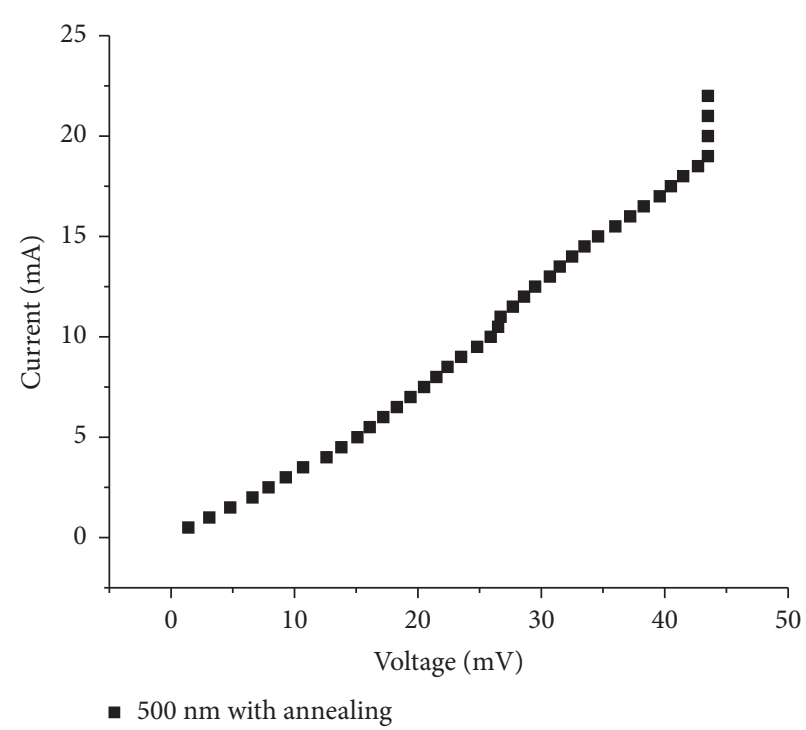

FIgURE 2: Dependence of voltage on current for a sample subjected to annealing at $100^{\circ} \mathrm{C}$.

merged with $\mathrm{Bi}$ and shows peak of $\mathrm{Bi}_{2} \mathrm{Te}_{3}$, as annealing temperature is increased. These peaks are visible by standard XRD Pro software. No additional peak has been observed after annealing which shows that the mixing due to annealing does not affect any structural face in comparison to asdeposited $\mathrm{Bi}_{2} \mathrm{Te}_{3}$ thin films. The intensity of individual peaks has been found to be increased after annealing which may be due to the fact that after annealing the $\mathrm{Bi}_{2} \mathrm{Te}_{3}$ thin films are arranged in more crystalline order due to heat treatment [9].

3.2.1. SEM Analysis. The effect of annealing on the morphological properties of the thin film has been characterized 


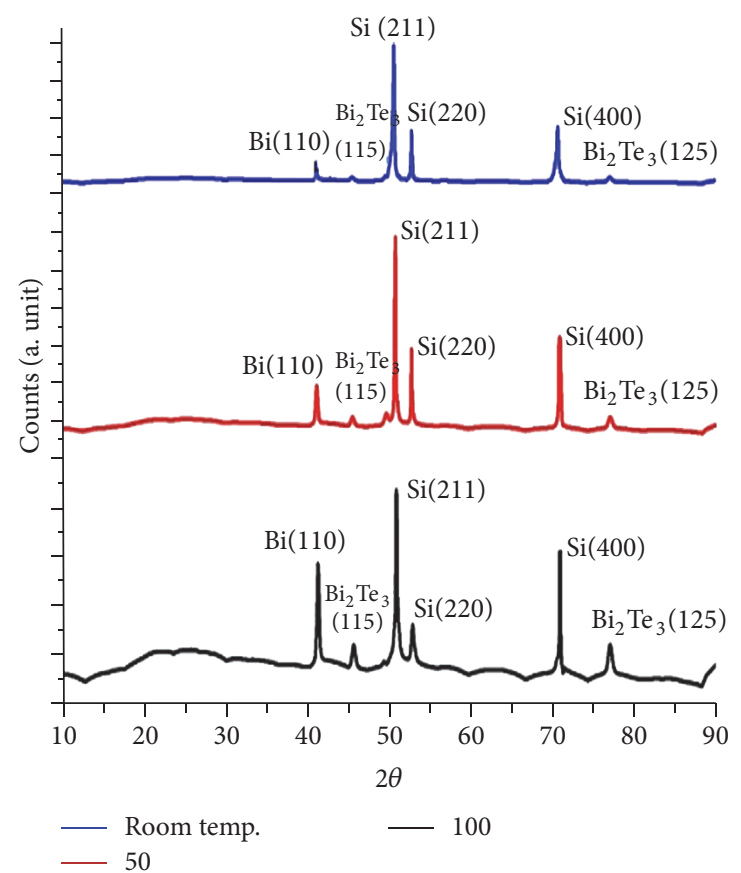

FIGURE 3: XRD for thickness of $500 \mathrm{~nm}$ at room temperature, annealed at $50^{\circ} \mathrm{C}$ and $100^{\circ} \mathrm{C}$.

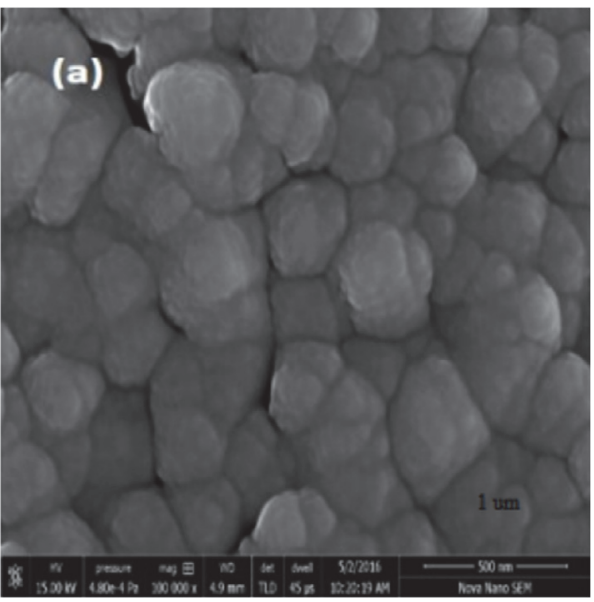

(a)

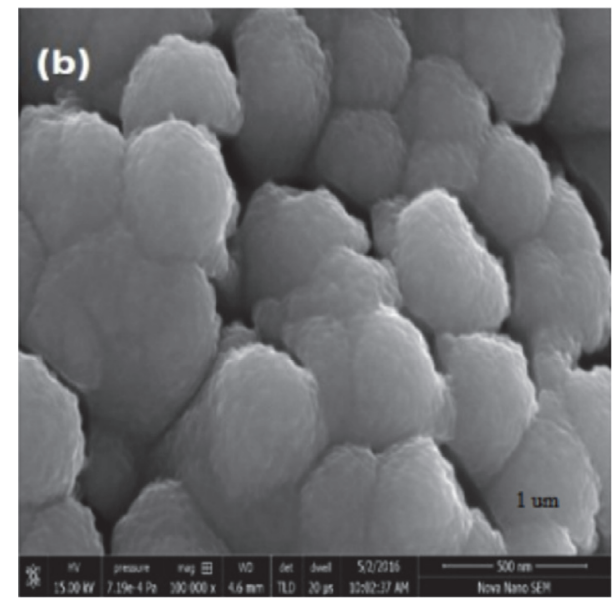

(b)

FIGURE 4: SEM images: (a) without annealing and (b) with annealing.

by recorded SEM images of thin films with or without annealing for different magnification increases from $10 \mathrm{um}$ to $1 \mathrm{um}$. These thin films clearly indicate that the tellurium and bismuth grow on Si substrate in spherical granular shape and the large granular clusters have appeared as the magnification increases [10].

The effect of annealing has been shown in Figure 4, which clearly indicates that without annealing thin film was smoother in surface morphology but after annealing there are tiny gaps that have appeared between the granules which may be due to diffusion of tellurium in bismuth which also confirms the mixing of deposited materials after annealing and supports our electrical studies.

\section{Conclusion}

It was observed that the thin film of bismuth and tellurium deposited on the silicon substrate without any heat treatment $V-I$ curves shows typical linear behaviour without any junction effect of different thickness at room temperature. Structural properties are investigated by X-ray diffraction and scanning electron microscopy (SEM) to check the crystallinity and granularity of the thin film. Without annealing, thin film was smoother in surface morphology and it shows the typical metallic nature and shows linear conducting behaviour. After heat treatment (annealing) at $50^{\circ} \mathrm{C}$ and $100^{\circ} \mathrm{C}$, it is observed that the $V$ - I curves show 
typical nonlinear curves due to granular uniformity of the surface of thin film. Grain growth has formed at the interface of bismuth and tellurium. Thin film indicates that after annealing there are tiny gaps that have appeared between the granules which may be due to diffusion of tellurium in bismuth and also confirms their mixing after annealing so the thin films attained the semiconductor nature and nonlinear behaviour.

\section{Conflicts of Interest}

The authors declare that they have no conflicts of interest.

\section{References}

[1] H. J. Goldsmid, Introduction to Thermoelectricity, chapter 1, Springer, Berlin, Germany, 2010.

[2] R. Setnescu, I. Bancuta, T. Setnescu, V. Cimpoca, S. Jipa, and I. V. Popescu, "Thermal characterization of semiconductor Bi2Te3 materials using DSC," Journal of Science and Arts, vol. 1, no. 12, pp. 95-102, 2010.

[3] M. Eginligil, W. Zhang, A. Kalitsov, X. Lu, and H. Yang, “Tunneling behavior of bismuth telluride nanoplates in electrical transport," Chemical Physics Letters, vol. 546, pp. 125-128, 2012.

[4] S. Gupta, S. Neeleshwar, V. Kumar, and Y. Y. Chen, "Synthesis ofbismuth telluride nanostructures by refluxing method," Advanced Materials Letters, vol. 3, no. 1, pp. 50-54, 2012.

[5] D. Jeffrey, M. Baodong, W. Junwei, D. Steven, and B. Clemens, "Effect of sintering on the thermoelectric transport properties of bulk nanostructured $\mathrm{Bi}_{0.5} \mathrm{Sb}_{1.5} \mathrm{Te}_{3}$ pellets prepared by chemical synthesis," Journal of Electronic Materials, vol. 41, no. 6, pp. 1408-1413, 2012.

[6] F. S. Bahabri, "Investigation of the structural and optical properties of bismuth telluride (Bi2Te3) thin films," Life Science Journal, vol. 9, pp. 290-294, 2012.

[7] H. M. A. Soliman and A.-H. B. Kashyout, "Electrochemical deposition and optimization of thermoelectric nanostructured bismuth telluride thick films," Engineering, vol. 3, no. 6, pp. 659667, 2011.

[8] W. Brostow, T. Datashvili, H. E. H. Lobland et al., "Bismuth telluride-based thermoelectric materials: coatings as protection against thermal cycling effects," Journal of Materials Research, vol. 27, no. 22, pp. 2930-2936, 2012.

[9] W. Chonkaew, W. Minghvanish, U. Kungliean, N. Rochanawipart, and W. Brostow, "Vulcanization characteristics and dynamic mechanical behavior of natural rubber reinforced with silane modified silica," Journal of Nanoscience and Nanotechnology, vol. 11, no. 3, pp. 2018-2024, 2011.

[10] G. H. Michler and F. J. Balta-Calleja, Materials: Introduction and Applications, Hanser, Munich, Germany, 2012. 

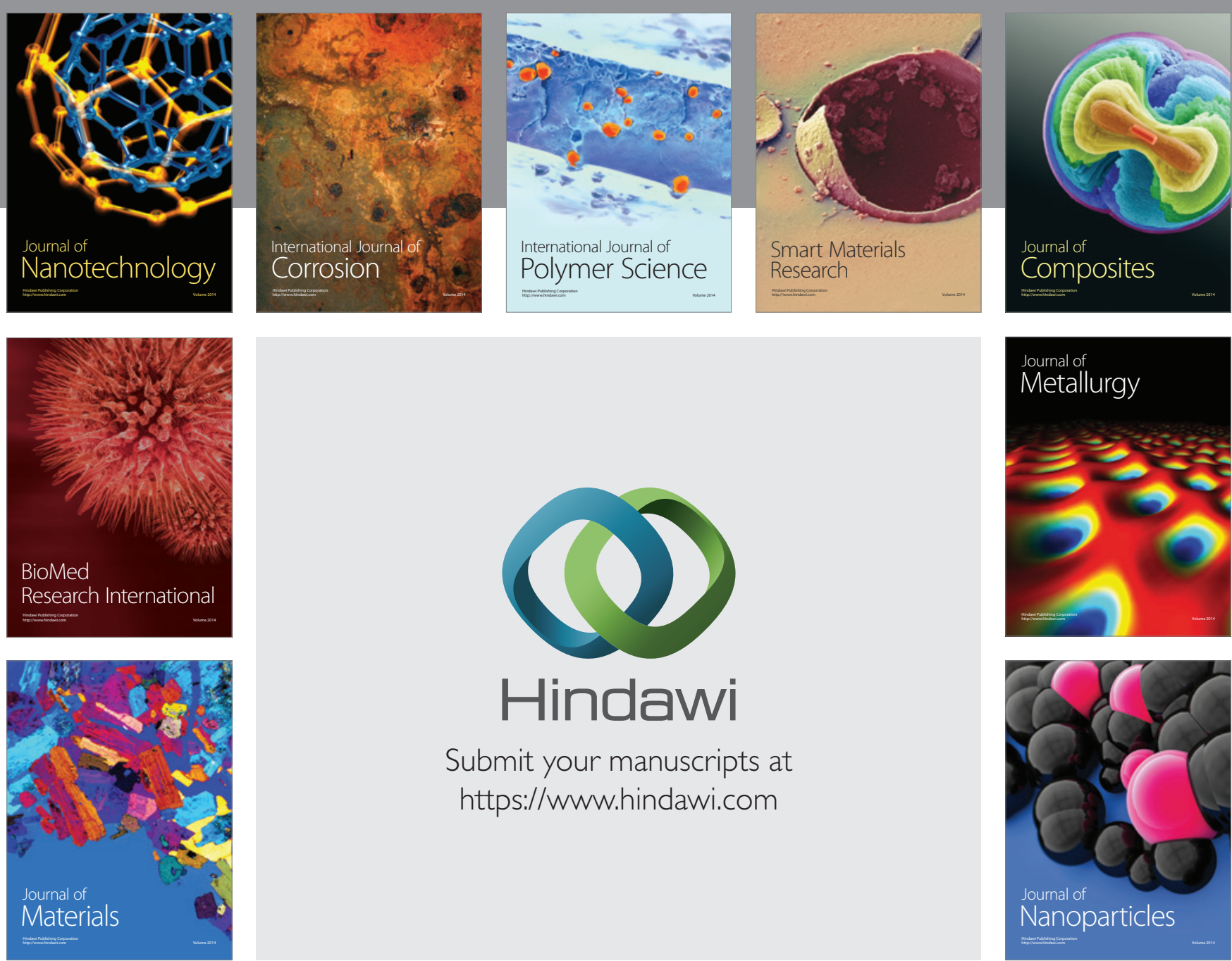

\section{Hindawi}

Submit your manuscripts at

https://www.hindawi.com

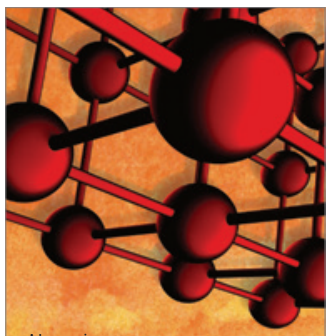

Materials Science and Engineering
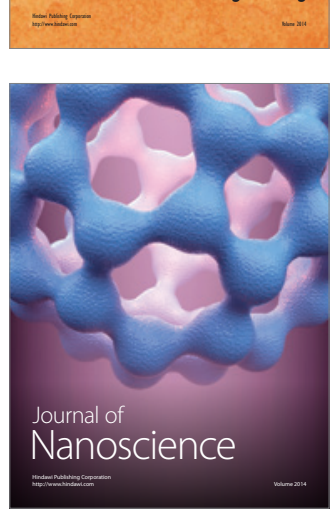
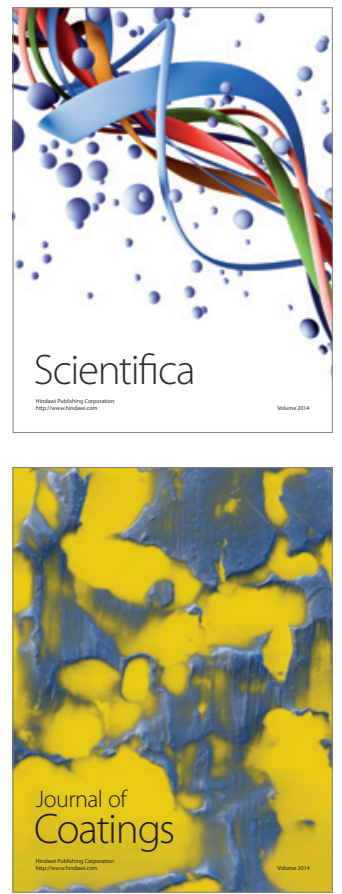
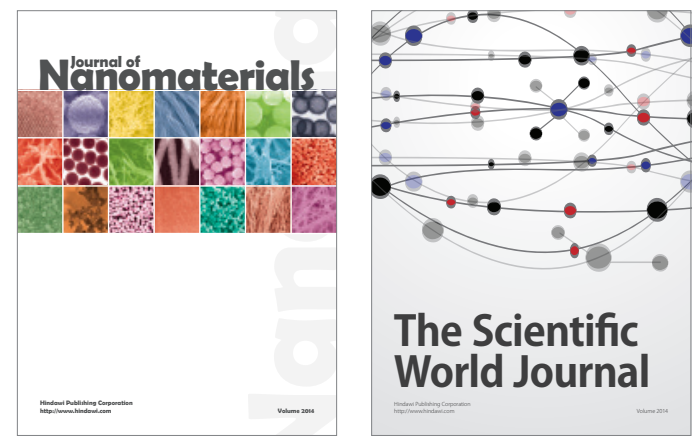

The Scientific World Journal
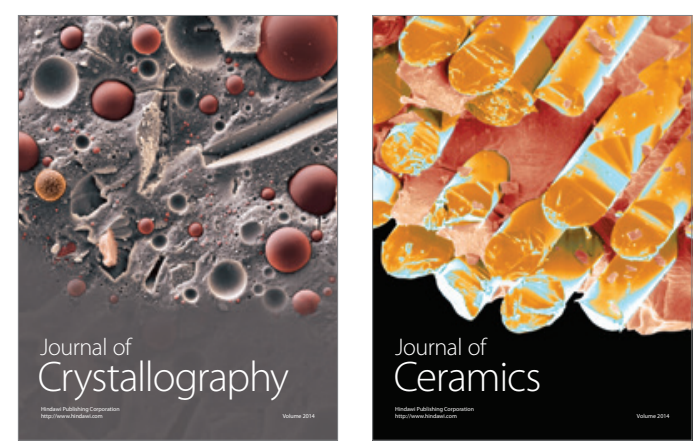
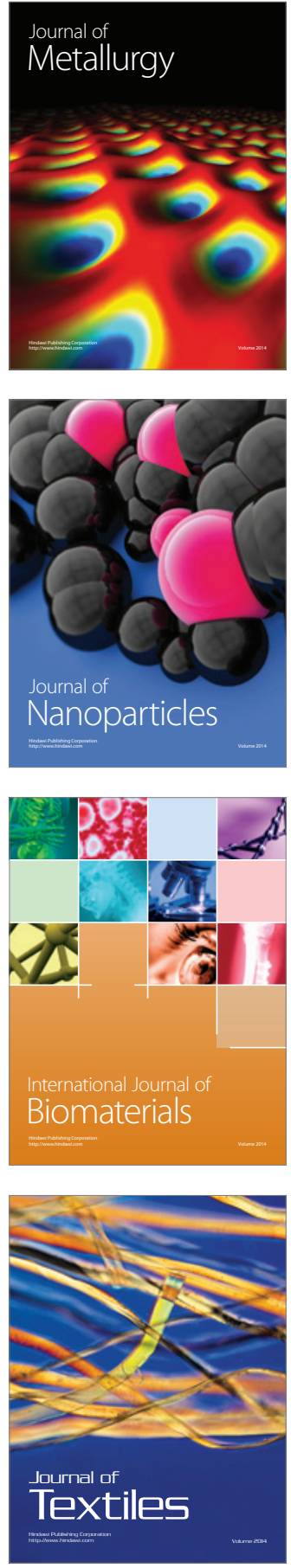